Check for updates

Cite this: RSC Adv., 2019, 9, 28657

\title{
Design of ultrathin hybrid membranes with improved retention efficiency of molecular dyes $\uparrow$
}

\author{
Peng Liu, ${ }^{a}$ Charles Milletto, ${ }^{a}$ Susanna Monti, (D) *b Chuantao Zhu ${ }^{a}$ \\ and Aji P. Mathew (D) *a
}

Ultrathin layers of 2,2,6,6-Tetramethyl-1-piperidinyloxy (TEMPO) Oxidized Cellulose Nanofibers (TOCNF) embedded with Graphene Oxide nanosheets (GOS) in different ratios were built, via the blade coating technique, on a polyvinylidene difluoride (PVDF) substrate to obtain superior membranes for separating water pollutants from aqueous media. Cellulose nanofiber-graphene oxide hybrid materials have shown a great potential for water purification due to their active microporous structure with extended areas rich in negatively charged carboxyl functional groups capable of adsorbing positively charged contaminants efficiently. In contrast to the pristine free-standing TOCNF films, which are completely impermeable, the ultrathin (68 nm thick) hybrid coating with a 100:1 TOCNF:GO ratio showed a stable water permeability $\left(816 \pm 3.4 \mathrm{~L} \mathrm{~m}^{-2} \mathrm{~h}^{-1} \mathrm{bar}^{-1}\right)$ higher than that of common polymeric membranes, and a very efficient size selectivity during filtration of water contaminated by various types of dyes. The membranes had high retention efficiency (82-99\%) for dyes with hydrated radii greater than $\approx 0.5 \mathrm{~nm}$ due to the favorable combination of electrostatic/hydrophobic interactions with the hybrid matrices and steric entrapment controlled by the pore size. This was confirmed by theoretical calculations that revealed both the structure and dynamic behavior of the dyes in the complex environment of the membranes.

Received 12th June 2019

Accepted 20th August 2019

DOI: $10.1039 / c 9 r a 04435 c$

rsc.li/rsc-advances cosmetics, leather, and textiles sectors to name just a few, because dyes are part of the chemicals used to impart special properties to their products. ${ }^{1,2}$ Dyes are usually classified into anionic, cationic and non-ionic (neutral) molecules, are stable to light and resistant to biological degradation and aerobic digestion. Although chemical, physical, and biological methods are frequently used to remove dyes form industrial effluents, adsorption is considered the cheapest and most effective method compared to the all the available dye removal strategies. ${ }^{3}$ Adsorbents as activated carbon are efficient in dye removal, but their use is limited because of their extremely high cost. ${ }^{4}$ Purification based on membranes is another advanced energy proficient technology that allows dye removal from water in continuous operation with efficiency close to $99 \%$ under optimised conditions of $\mathrm{pH}$ and concentration. ${ }^{5}$ The major industrial challenges in membrane technology are, however, high cost, fouling and limited flux. According to Hagen-Poiseuille's equation, the permeation flux is inversely proportional to the total distance of the liquid running through the membrane. ${ }^{6,7}$ Ideal membranes are, therefore, expected to have very thin film barriers with the appropriate pore size to maximize permeation flux at low pressure and obtain an excellent dye retention. Recently, a new type of thin-film nanocomposite membrane (TFN) containing ultrathin barriers with good separation efficiency have been developed by incorporating suitable nanofillers (e.g. nanofibers, nanoparticles and nanosheets) into the top selective layers of the porous support. ${ }^{8-11}$

${ }^{a}$ Division of Materials and Environmental Chemistry, Stockholm University, Stockholm, 10691, Sweden.E-mail: aji.mathew@mmk.su.se

${ }^{b}$ CNR-ICCOM, Institute of Chemistry of Organometallic Compounds, Via G. Moruzzi 1, I-56124 Pisa, Italy. E-mail: sapeptides@gmail.com

$\dagger$ Electronic supplementary information (ESI) available: Tables S1 and S2; Fig. S1-S6. See DOI: 10.1039/c9ra04435c 
The focus has, indeed, moved on to layered adsorption membranes made of nanoscaled adsorbents as zeolites, MOFs, graphene oxide and other compounds. ${ }^{\mathbf{1 2 - 1 9}}$ Considering, for example, nanocellulose based materials, inspired by the concept of thin-film nanocomposites, both Cao et al. ${ }^{\mathbf{2 0}}$ and Ma et $a l .{ }^{9}$ introduced into the membranes thin TOCNF layers by casting and impregnating electrospun mats, which were prepared and characterized for ultrafiltration with pore sizes in the 10-100 nm range..$^{9,20}$

TEMPO-oxidised cellulose nanofibers (TOCNF) have been established as highly functional and biobased adsorbents for water purification due to their high specific surface area, abundant functionality, high strength and crystallinity. ${ }^{21-30}$ Moreover, TOCNF with small diameters $(>10 \mathrm{~nm})$ and high aspect ratios $(>100)$ represent an ideal 1D nanomaterial for the fabrication of ultrathin films..$^{21,23,31}$

In this work, we have combined TOCNF and GO to create thin film membranes with superior performance and here we report the results obtained by investigating the homogeneous deposition of TOCNF-GO ultrathin films (thickness $<100 \mathrm{~nm}$ ) on a polyvinylidene difluoride (PVDF) microporous substrate (which was widely used for membrane distillation since it is intrinsically hydrophobic ${ }^{32}$ ) by the blade coating technique. As already observed, GO nanosheets are potential candidates for membrane barrier layers due to their unique 2D mono-atomic thick asymmetrical structure and the oxygen-functional groups on the edges of the basal plane. In addition, GO nanosheets have a high chemical resistance and can be easily dispersed and processed in liquid dispersions. ${ }^{33-35}$ Both GO and TOCNF have excellent compatibility and are connected through relatively strong intermolecular interactions. ${ }^{36-38}$ This was revealed by Zhu and coworkers who successfully prepared self-assembled TOCNF-GO biohybrids made of intermixed GO nanosheets and TOCNF with promising adsorption capacity towards $\mathrm{Cu}^{2+}$ ions. ${ }^{25}$

The doctor blade coating technique is easily scalable and can be industrially adapted for the fabrication of membranes with a fine control of the coating thickness. The contribution of the adsorption and size exclusion phenomenon by the membranes was studied focusing on selected anionic, cationic and neutral dyes, namely Victoria Blue B (VB), Rhodamine B (RB), Methyl Orange (MO), Rhodamine 6G (R6G), Methyl Blue (MB) and Methylene Blue (MnB) and correlated to membrane charge, composition and morphology. To support and interpret the experimental findings, quantum chemistry calculations combined with a classical conformational search procedure and reactive molecular dynamics simulations in the complex environment of a membrane were performed to disclose the dye structure and dynamics. The characteristics of the dyes that could be correlated with their entrapment, interaction with the matrices and motion in solution were analysed and linked with the experimental evidence.

\section{Experimental}

\subsection{Materials}

We prepared 2,2,6,6-tetramethyl-1-piperidinyloxy (TEMPO) mediated oxidised cellulose nanofibers (TOCNF) by dissolving cellulose fibers (Domsjö Fabriker AB, Örnskoldsvik, Sweden) according to the previously reported method by Saito et al. ${ }^{39}$ The cellulose pulp was firstly mechanically beaten, followed by TEMPO mediated oxidation (10 $\mathrm{mmol}$ hypochlorite per gram of pulp) and finally disintegration via homogenization. A water suspension of TEMPO CNF at 0.4 wt $\%$ was obtained after multiple-pass homogenization (two passes), using an APV 2000 homogeniser. Graphene oxide (GO) dispersion in water with a concentration of $4 \mathrm{mg} \mathrm{mL}^{-1}$ was purchased from SigmaAldrich (Sweden). Copper(II) nitrate hydrate, calcium chloride hydrate, cadmium nitrate hydrate, lead(II) nitrate, Victoria Blue $\mathrm{B}(\mathrm{VB}$, positive charge at $\mathrm{pH} 5.6)$, Rhodamine $\mathrm{B}(\mathrm{RB}$, neutral at $\mathrm{pH}$ 4.7), Methyl Orange (MO, negative charge at $\mathrm{pH} 5.8$ ), Rhodamine 6G (R6G, positive charge at $\mathrm{pH}$ 3.9), Methyl Blue ( $\mathrm{MB}$, negative charge at $\mathrm{pH}: 6$ ) and Methylene Blue (MnB, positive charge at $\mathrm{pH}$ 7.2) were all purchased from SigmaAldrich, Germany and used as received.

\subsection{Preparation of dispersions containing GO and TOCNF}

The dispersions of GO and TOCNF were mixed using sonication (Q500 Sonicator, Qsonica) in deionized water for 5 minutes with different GO : TOCNF mass ratios (Table S1, ESI $\dagger$ ). The concentration of TOCNF in all dispersions were the same (4 $\mathrm{mg} \mathrm{L}^{-1}$ ), and combined with GO in mass ratios of $1: 100$ $\left(\right.$ TOCNF-GO ${ }_{1}$ ), $5: 100\left(\right.$ TOCNF-GO ${ }_{5}$ ) and $10: 100$ (TOCNF$\left.\mathrm{GO}_{10}\right)$.

\subsection{Preparation of TOCNF-GO thin film nanocomposite membranes}

The TOCNF-GO membranes were prepared using a film applicator (Elcometer K4340M101 motorised film applicator, Elcometer Instruments, UK). The doctor blade gap width $\left(h_{0}\right)$ could be varied in the $10-200 \mu \mathrm{m}$ range and the coating processing speed $\left(v_{0}\right)$ in the $0.5-10 \mathrm{~cm} \mathrm{~s}^{-1}$ range. The optimum $h_{0}$ and $v_{0}$ for the fabrication of TOCNF-GO membranes were investigated. TOCNF ( $4 \mathrm{mg} \mathrm{L}^{-1}$ ) and TOCNF-GO dispersions were coated on the porous polyvinylidene difluoride (PVDF) substrate (pore size: $0.45 \mu \mathrm{m}$, HVLP04700, Durapore Membrane Filter). After wet coating, all the membranes were dried overnight at ambient conditions.

\subsection{Characterisation}

2.4.1 Morphological analysis. The morphology of TEMPOmediated oxidised nanofibers and graphene oxide nanosheets were studied with a Fast Scan Atomic Force Microscope (AFM, Bruker, Nanoscope controller, Santa Barbara, California, USA). In all cases, the height sensor, peak force error images were recorded using the probe (Model: ScanAsyst-air, Bruker) under the Peak Force tapping mode. The collected data were processed with NanoScope Analysis 1.5 software (Bruker). The morphology of the membranes was studied using a field emission Scanning Electron Microscope (SEM, JSM-7401F, Japan) after sputter coating (JEOL, JFC-1200) with gold for 30 seconds. The cross section was then imaged using the scanning electron microscope (JSM-7401F) operating at a low voltage (1 or $2 \mathrm{kV}$ ) using the side mounted secondary electron detector. 
2.4.2 Surface charge content and zeta potential. Surface charge content of TOCNF and GO nanosheets was determined by the conductivity titration method. The zeta potentials of TOCNF and GO at different $\mathrm{pH}$ were measured using a Zeta sizer nano ZS, Malvern (UK) at $25{ }^{\circ} \mathrm{C}$. The concentrations of all the measured samples were $0.05 \%$ wt.

2.4.3 Rheological measurements. A Physica MCR 301 rheometer (Anton Paar, Austria) was used to measure the viscosity of the dispersions. A titanium coated cone-plate with a $60 \mathrm{~mm}$ diameter and a cone angle of $1^{\circ}$ was used. During the measurements temperature was kept constant at $22{ }^{\circ} \mathrm{C}$. The experiments were performed using $2 \mathrm{ml}$ dispersions of TOCNF (4 $\mathrm{g} \mathrm{L}^{-1}$ ), TOCNF-GO membranes with a constant gap of 0.05 $\mathrm{mm}$.

2.4.4 Water permeability. The water permeability of the membranes was examined using a commercial bench-scale stainless steel dead-end stirred cell-filtration unit (Sterlitech HP4750 High Pressure Stirred Cell, USA). The effective membrane area was $14.6 \mathrm{~cm}^{2}$ and all the experiments were performed at ambient conditions with a compressed air pressure of 2.2 bar. The volume of water that passed through the membrane for a definite time interval was measured and the flux was calculated in standard units of $\mathrm{L} \mathrm{m}^{-2} \mathrm{~h}^{-1} \mathrm{bar}^{-1}$. Each measurement was repeated consecutively three times with the same membrane.

2.4.5 Dye removal by the membranes. The retention (\%) and solution flux $\left(\mathrm{L} \mathrm{m}^{-2} \mathrm{~h}^{-1} \mathrm{bar}^{-1}\right)$ of the membranes for the dyes were examined using the same dead-end stirred cell unit that was used for the water permeability measurement. To minimize the polarization effect due to the concentration on the retention and flux performance, the membranes were evaluated by filling the cell with $30 \mathrm{ml}$ of dye solution followed by the exertion of a constant pressure (2.2 bar) to the membrane for inducing the permeation of a portion $(6 \mathrm{ml})$. The flux of the dye solution was measured and calculated similarly to the water flux measurement. ${ }^{34}$ The quantitative analysis of dye concentration in the permeate was determined by a UV-vis spectrophotometer (PerkinElmer, Lambda 2S, Sweden). The absorbance of each dye was recorded at their $\lambda_{\max }$ (Table S2, ESI $\dagger$ ).

\subsection{Molecular modeling}

2.5.1 Conformational search. To build a set of representative conformers of the six dyes, namely Victoria Blue $\mathrm{B}(\mathrm{VB}, \mathrm{pH}$ : 5.6), Rhodamine B (RB, pH: 4.7), Methyl Orange (MO, pH: 5.8), Rhodamine 6G (R6G, pH: 3.9), Methyl Blue (MB, pH: 6) and Methylene Blue (MnB, pH: 7.2), first we checked the Cambridge Structural Database and downloaded the available structures, then we built the other ones and optimized all of them at the quantum chemistry level. Then, we used the Balloon molecular conformation engine, ${ }^{\mathbf{4 0 , 4 1}}$ which is capable of exploring efficiently the energetic landscape of the molecules and is freely downloadable form the internet (http://users.abo.fi/mivainio/ balloon/index.php), to identify other possible structures. Briefly, Balloon contains a conformational search engine that is based on the distance geometry approach and on multiobjective genetic algorithms, which change the torsional angles of the molecules, the stereochemistry of double bonds, the tetrahedral chiral centers and ring conformations. Modifications of the geometries are accompanied by short energy minimizations that are based on a re-implementation of the MMFF94 molecular force field. These are essentially used to remove unrealistic structures. A preliminary exploration of the best searching conditions was performed to select the most appropriate values of the parameters responsible for the conformational search procedure (considering the number of identified geometries, the energy of the lowest conformers and the number of times their structures were visited). The check suggested that the lowest energy structures were, indeed, the global minima of each molecule and the created ensembles could indeed represent them on the MMFF94 potential energy surface. Balloon was run for 200 generations on each molecule using an initial population size of 50 conformers. The rings were treated as frozen fragments and default values were chosen for the probabilities of the genetic operators. The maximum number of conjugate gradient geometry optimization steps and the convergence criterion based on the gradient root-mean-square (rms) were set to 500 and $0.01 \mathrm{kcal} \mathrm{mol}^{-1} \AA^{-1}$, respectively. The EEM charge model and the full force field in post-GA optimization of the conformers were employed. It is evident from the examination of all the molecular structures that they have a limited flexibility. All the conformations identified by the Balloon conformational search were then optimized at the quantum chemistry (QC) M06-2X/6-311G(d,p) level of theory in the gas phase by means of Gaussian09 revision A02 (ref. 42) and used to estimate the radius of gyration and characteristic molecular electrostatic potential maps, which could be useful to disclose typical adsorption modes of the molecules on/inside the substrates. As far as the functional is concerned, M06-2X, which has an s6 scaling factor of Grimme's long-range dispersion correction of only 0.06 , can be considered an appropriate choice because, as demonstrated in the literature, it can describe dispersion interactions for neutral systems and predict structures and relative energies of different types of neutral and charged molecules.

2.5.2 Hydrated radius of the dyes. To estimate the average hydrated radius of the dyes their minimum energy conformations, identified during the conformational search, were used to calculate the solvent-accessible surface area (SASA) which was generated considering a sphere with a probe radius of $1.4 \AA$. Then, from the surface areas, as done by Akbari and coworkers, ${ }^{43}$ an "equivalent spherical radius" was calculated. The linear correlation between the SASA radii and the known hydrated radii (Table S2, ESI $\dagger$ ), was satisfactory having a $\chi^{2} \approx$ 0.95 , which was used to estimate the hydrated radius of the VB dye, which turned out to be $6.3 \AA$ (Table S2, ESI $\dagger$ ).

2.5.3 Molecular electrostatic potential (MEP). Electrostatic interactions play a key role in molecular recognition and binding and the view of the molecular electrostatic potential around a molecule can be very useful to analyse them. ${ }^{\mathbf{4 4 , 4 5}}$ Indeed, the MEP was used to disclose substituent behavior, to identify nucleophilic and electrophilic sites, to reveal intermolecular arrangements and adsorption modes on different types of interface, ${ }^{46}$ to examine the substituent effect on various 
molecular systems. ${ }^{47}$ The MEP of the selected dyes (minimum energy conformation) was calculated at the quantum chemistry level used for optimizing their structures (see computational details above) and visualized on the solvent accessible surface. Negative, positive and zero potential regions are shown in red, blue and white, respectively, while fading colors are used to represent decreasing values. In the case of charged systems, depending on the sign, the MEP was positive or negative everywhere (Fig. S2 $\dagger$ ). Thus, it can be speculated that, in principle, the first step of the recognition/binding/adsorption process was a migration towards the regions of the substrate with the opposite sign (favourable attraction). However, the surrounding environment induced a redistribution of the total charge of the dye and determined its preferential orientation and adsorption mode. In order to gain a clearer view of the local behaviour of the dyes, it was necessary to unmask the charge effect by neutralizing the total charge of the molecule. This was achieved by simulating a sort of interaction with the surrounding species by adding or removing hydrogen atoms (hypothesizing possible exchanges between the dye and the GOTOCNF support).

2.5.4 Adsorption of selected dyes on the cellulose-based supports. In order to reveal possible binding modes of the dyes on the hybrid GO-TOCNF support a model made of a nanocellulose fibril modified through position selective catalytic oxidation of the C6 primary hydroxyls exposed to the solvent (TOCNF) $)^{\mathbf{2 1 , 4 8}}$ combined with a monolayer of GO sheets of various size was prepared on the basis of the experimental observations and used to investigate the behavior of two representative dyes, namely a cationic (VB) and an anionic (MO) species.

2.5.5 Cellulose-based support model. The starting configuration of the hybrid bio-sorbent model was built as a layered membrane with three GO sheets (corresponding to an almost total coverage) placed close to the TOCNF matrix in parallel orientation (flat adsorption). TOCNF consisted of sixteen chains of sixteen glucosyl residues each, arranged as a parallelepiped rod. Only one facet was functionalized with carboxyl groups and a realistic length was obtained by replicating the system in the $x$ and $y$ direction (the fibril size was around $84 \times 25 \times 25 \AA^{3}$ ). The chosen size was sufficient to simulate the variability of the environment and represent reasonably the experimental observations at an affordable computational cost. Representative configurations of GO (with 28 and 51 carbon rings) were downloaded from the Automated Topology Builder (ATB) and Repository site (http://atb.uq.edu.au/molecule.py? molid=34774) and modified by adding rings and edge carboxyl groups. ${ }^{\mathbf{4 9 5}}$ The molecules were optimized at the quantum chemistry DFT/M062X/6-31G(d) level and used as starting geometries in all the simulations. Before creating the complete systems with cellulose, dyes and solvent molecules, the substrate model was optimized and equilibrated in solution by means of short atomistic molecular dynamics (MD) simulations based on a reactive force field (ReaxFF approach) appropriately parametrized to describe these kinds of system. ${ }^{51-56}$ Preequilibration was performed in the NPT ensemble, at $300 \mathrm{~K}$ and $1 \mathrm{~atm}$, to prepare the supports for subsequent simulations in crowded environments.
2.5.6 Molecular dynamics simulations. Two multicomponent configurations consisting of the equilibrated GO-TOCNF model, dye molecules, water and counterions $\left(\mathrm{Cl}^{-}\right.$or $\mathrm{Na}^{+}$ depending on the charge of the dye), were prepared and simulated for hundreds of picoseconds until they reached their final equilibrium. The most crowded scenario contained about ten thousand atoms. No restraints were introduced in the systems and reactivity was always on to take into account the protonation and deprotonation of the various groups in response to the surrounding environment. All the MD simulations were obtained with the Amsterdam Density Functional (ADF)/ReaxFF software (ReaxFF release adf2017.114). ${ }^{57}$ The systems were first equilibrated in the NVT ensemble and then at constant pressure and temperature $(T=300 \mathrm{~K}, P=1 \mathrm{~atm})$ for about $300 \mathrm{ps}$. Subsequently, the production dynamics were performed in the NVT ensemble for about 1 ns and system structures were collected every 0.1 ps. Temperature and pressure were controlled through the Berendsen's thermostat and barostat with relaxation constants of $0.1 \mathrm{ps}$ and the time step was set to 0.25 fs. Considering that we were interested in revealing the characteristic adsorption of the dyes on the substrate, and that the dyes were already near the support at the beginning of the simulations, the simulated production time span was substantial to reach the final settlement. Thus, the analysis of the sampled data was focused on the last portions of the trajectories, which were obtained from ten simulations with different starting orientations of the dyes in relation to the substrates (at a distance of about $8 \AA$ ). The final analysis was based on all of the sampled data and tried to depict and predict the various tendencies of dyes in connection with experiments. Examined descriptors were mainly atom-atom radial distribution functions (RDFs), spatial distribution functions (SDFs) and hydrogen bonds. However, visual inspection of the trajectories was fundamental to understand the adsorption scenarios and evidence the different adsorption behavior of the two dyes.

\section{Results and discussion}

\subsection{Characterizations of TOCNF and GO}

As displayed in Fig. 1-left, the diameters of the oxidised cellulose nanofibers were found to be in the 1-3 $\mathrm{nm}$ range and their lengths varied from several hundred nanometers to micrometers. As it can be noticed by inspecting Fig. 1-left, the thickness of the GO layer is rather uniform (around $1 \mathrm{~nm}$ ), whereas the lateral width is more variable, ranging from hundreds of nanometers to several micrometers, which is significantly larger than the diameter of the TOCNF fibers. Both of these nanoscale components had very high theoretical specific surface areas (SSA), which were in the range 888-2666 $\mathrm{m}^{2} \mathrm{~g}^{-1}$ in the case of TOCNF and approximately $2039 \mathrm{~m}^{2} \mathrm{~g}^{-1}$ for GO. The zeta potentials of both TOCNF and GO were tendentially negative and remained such in the whole $\mathrm{pH}$ range examined in this study. The most negative values were found at neutral $\mathrm{pH}$ (around 8), due to the high population of the acidic charged groups (carboxyl groups) that were present on both TOCNF and GO (Fig. 1-right). Inspecting the trends shown in the figure it is apparent that TOCNF had the highest negative zeta potential 

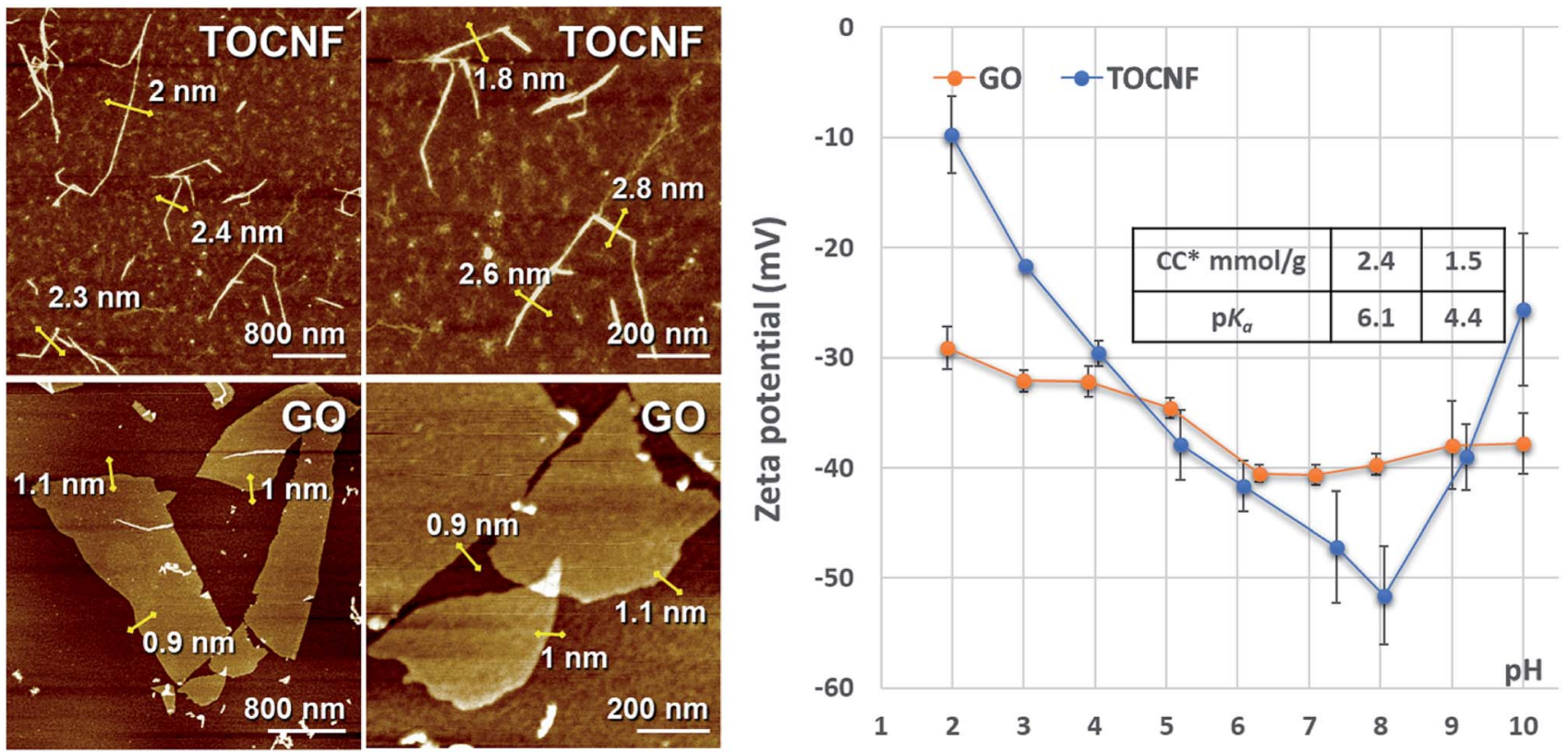

Fig. 1 Characterization of TOCNF and GO. (Left) Morphologies detected using AFM; (Right) charge contents (CC), pK $\mathrm{a}$ of the acidic functional groups and the zeta potentials as a function of $\mathrm{pH}$.

$(-51 \mathrm{mV})$ at neutral $\mathrm{pH}$, whereas at $\mathrm{pH}=10$ the zeta potential became less negative $(-26 \mathrm{mV})$ and it was further reduced to $-9.7 \mathrm{mV}$ at $\mathrm{pH}=2$. These sensitive variations were not observed, instead, in the case of GO where the explored zeta potential range was narrower (only $9 \mathrm{mV}$ compared to $41 \mathrm{mV}$ ), the minimum and maximum values were found at $\mathrm{pH}=10$ $(-38 \mathrm{mV})$ and $\mathrm{pH}=1.9(-29 \mathrm{mV})$, respectively.

\subsection{Rheological characterizations of TOCNF-GO mixed dispersions}

Considering that rheological properties of dispersions are crucial when the blade coating technique is used for fabricating the membranes, they were examined in detail and presented qualitatively, as photographs (Fig. 2-top) and quantitatively, through the plot of the shear viscosity as a function of the shear rate (Fig. 2-bottom).

The images (Fig. 2-top) clearly suggest that both GO and TOCNF formed stable dispersions at various mass ratios, because of the repulsive interactions resulting from the highly charged surfaces. The flow birefringence photos demonstrate that the dispersions of GO nanosheets and TOCNF nanofibers resulted in stable colloidal systems without any macroscopic aggregates and had a tendency to align in a chiral nematic liquid crystal phase. Indeed, flow birefringence is the double refraction of light that passes through a transparent, molecularly ordered medium and arises from the orientationdependent differences in the refractive index originated in various regions within the dispersion. As shown in Fig. 2-top, TOCNF $\left(4 \mathrm{~g} \mathrm{~L}^{-1}\right)$ was a gel-like substance almost transparent, whereas the TOCNF- $\mathrm{GO}_{10}$ dispersion, which contained the highest GO concentration, was dark brown and significantly less viscous than the other samples. It can also be noted that the
TOCNF suspension showed the presence of a birefringent glassy phase (remarkable birefringence without flow) with high birefringence intensity (which could be deduced from the whiteness of the areas), whereas the flow birefringence patterns observed in the GT dispersions (especially at higher GO concentrations) displayed some birefringent oriented elliptical domains of a few millimeters with a remarkable distinction direction. However, the different alignments of the individual domains suggest that the global distribution of the orientations is random. Birefringence occurred in the whole area of the dispersions.

As is evident from Fig. 2-bottom, the TOCNF-GO dispersions displayed non-Newtonian shear-thinning (pseudoplastic) behavior, indicating the formation of a network structure between two GO and TOCNF components. This was realized through hydrogen bonding interactions between the hydroxyl, epoxy, carboxyl groups and oxygen species available on both interfaces. The marked decrease in viscosity of the TOCNF-GO dispersions with the increase in the shear rates is consistent with the rheological characteristics of both GO and TOCNF, ${ }^{58,59}$ whereas the drastic reduction of the shear viscosity with the raise in GO content at all shear rates could be ascribed to a weakening of the interactions between the GO nanosheets and the TOCNF fibers (most probably due to disruption of the intermolecular hydrogen bond network involving the carboxyl groups) and to a heterogeneous distribution of $\mathrm{GO}$ in the TOCNF matrices. Indeed, it could be speculated that GOTOCNF interactions prevailed over GO-GO self-aggregations at low GO content, favouring the formation of GO-TOCNF layered structures uniformly dispersed in the TOCNF matrix, whilst when the percentage of GO increased the GO self-interaction contribution became equally important and stacked GO aggregates could be found in various orientations in relation to the 

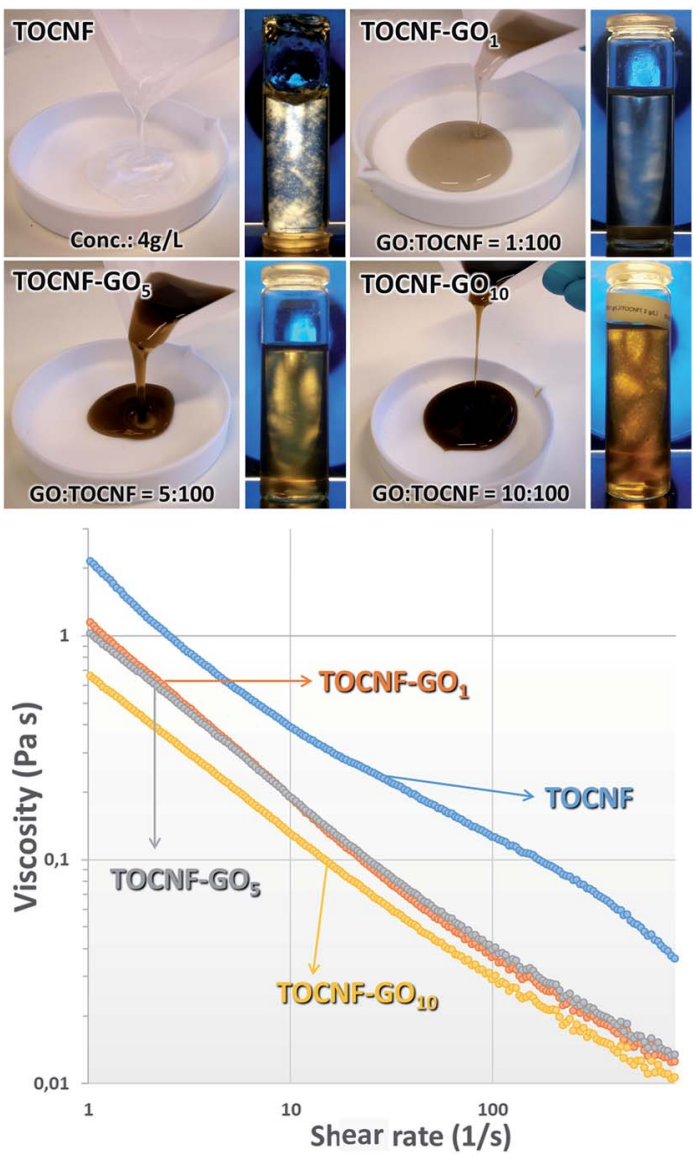

Fig. 2 Properties of TOCNF-GO dispersions (TOCNF content is $4 \mathrm{~g}$ $\mathrm{L}^{-1}$ for all the suspensions; the TOCNF : GO mass ratios are $100: 1$, $100: 5$ and $100: 10$ ). (Top) Photos of the suspension and all the dispersions flanked by the flow birefringence of the dispersions. (Bottom) Shear viscosity vs. shear rate $\left(\mathrm{s}^{-1}\right)$. Rheology for the pure TOCNF suspension and TOCNF-GO hybrid dispersions.

TOCNF fibers. This disorganization of the layers could induce a sort of partial collapse of the compact network structure, reorganization in a more amorphous $3 \mathrm{D}$ random arrangement, and thus an increase in flow with a consequential decrease in viscosity. ${ }^{25,60}$

\subsection{Membrane fabrication coating process}

The dispersions were coated on the porous substrate using the doctor blade coating technique as presented in the scheme depicted in Fig. S1. $\dagger$ The blade gap width $\left(h_{0}\right)$ and the coating speed $\left(v_{0}\right)$ are the two most vital processing parameters that have a substantial impact on the structure and, therefore, on the performance of the fabricated membrane. The blade gap width has a positive linear correlation with the coated film thickness and coating grammage. Thus, to investigate the optimal processing parameters, the performance of the TOCNF membrane as a function of $h_{0}$ and $v_{0}$ was evaluated by measuring the retention and solution flux when Victoria Blue $\mathrm{B}$ (VB), Rhodamine B (RB) and Methyl Orange (MO) dyes were present in solution (feed concentration of $10 \mathrm{mg} \mathrm{L}^{-1}$ ). The thickness, $d$, of the dried coated film was calculated from the empirical relationship: ${ }^{61}$

$$
d=\frac{1}{2}\left(h_{0} \frac{c}{\rho}\right)
$$

where $h_{0}(\mu \mathrm{m})$ is the blade gap width, $c$ is the concentration of the dispersion $\left(\mathrm{g} \mathrm{cm}^{-3}\right)$, and $\rho$ is the material density (the densities of GO and TOCNF are $0.981 \mathrm{~g} \mathrm{~cm}^{-3}$ and $1.46 \mathrm{~g} \mathrm{~cm}^{-3}$, respectively ${ }^{62}$ ). The optimum parameters identified in the preliminary investigations, namely blade gap width $h_{0}=50 \mu \mathrm{m}$ and coating speed $v_{0}=0.5 \mathrm{~cm} \mathrm{~s}^{-1}$, were used for the fabrication of the TOCNF-GO membranes. The nomenclature and processing parameters of the thin film nanocomposite membranes are reported in Table S1 (ESI). $\dagger$ Both photographs and SEM images (Fig. 3) revealed the homogeneity of the thin densely coated layer and the surface structure of the microporous substrate beneath it, which is partially visible in the SEM images (Fig. 3(d)). The image highlights the ultralow thickness of the coating and the tight contact between the coated layer and substrate. The surface structure of the TOCNF- $\mathrm{GO}_{1}$ membrane was similar to that of the pristine TOCNF one (Fig. 3(b)). The low grammage $\left(0.1 \mathrm{~g} \mathrm{~m}^{-2}\right)$ TOCNF- $\mathrm{GO}_{1}$ thin film coatings correspond to a coating thickness of approximately $68 \mathrm{~nm}$.

\subsection{Filtration performance}

Water permeability of the membranes with different GO dosages was tested through three consecutive filtrations of deionized water and the results are displayed in Fig. 4(a). The permeability of the TOCNF membrane decreased sharply and continuously from 1515 to $571 \mathrm{~L} \mathrm{~m}^{-2} \mathrm{~h}^{-1}$ bar $^{-1} .{ }^{21}$ Both the increase in film thickness and the narrowing of the water channels, due to nanofiber swelling, lead to the fast decrease in water permeability in line with the studies of Hakalahti and coworkers that justify this behavior considering the hydrophilic nature of the matrix, the abundance of water binding functional groups and the thickness of free-standing TOCNF films. ${ }^{63}$ On the one hand, TOCNF-GO coated membranes exhibited stable water permeability (Fig. 4), which showed that the added GO nanosheets could function as rigid skeletons inside the TOCNF matrix structure. This prevented swelling and contributed to the structural stability of the pores (Fig. 4(b)). On the other hand, a high amount of GO was detrimental for the permeability of water as they could narrow or even seal some water channels. Indeed, when the GO mass ratio increased from 1 to 10 , water permeability dropped sharply from 812 to $82 \mathrm{~L} \mathrm{~m}^{-2} \mathrm{~h}^{-1} \mathrm{bar}^{-1}$. Among the examined membranes, the TOCNF- $\mathrm{GO}_{1}$ one had the best water permeability performance with a good balance between mechanical stability and high water permeation. It exhibited an incomparably high permeability in relation to previous studies as well, due to its extremely low thickness (68 $\mathrm{nm}$ ) and swelling resistant structure. According to the HagenPoiseuille equation, the permeation flux is inversely proportional to the permeation distance, implying that a separation layer should be as thin as possible to maximize the flux. ${ }^{7}$ Freestanding TOCNF films traditionally prepared through vacuum 

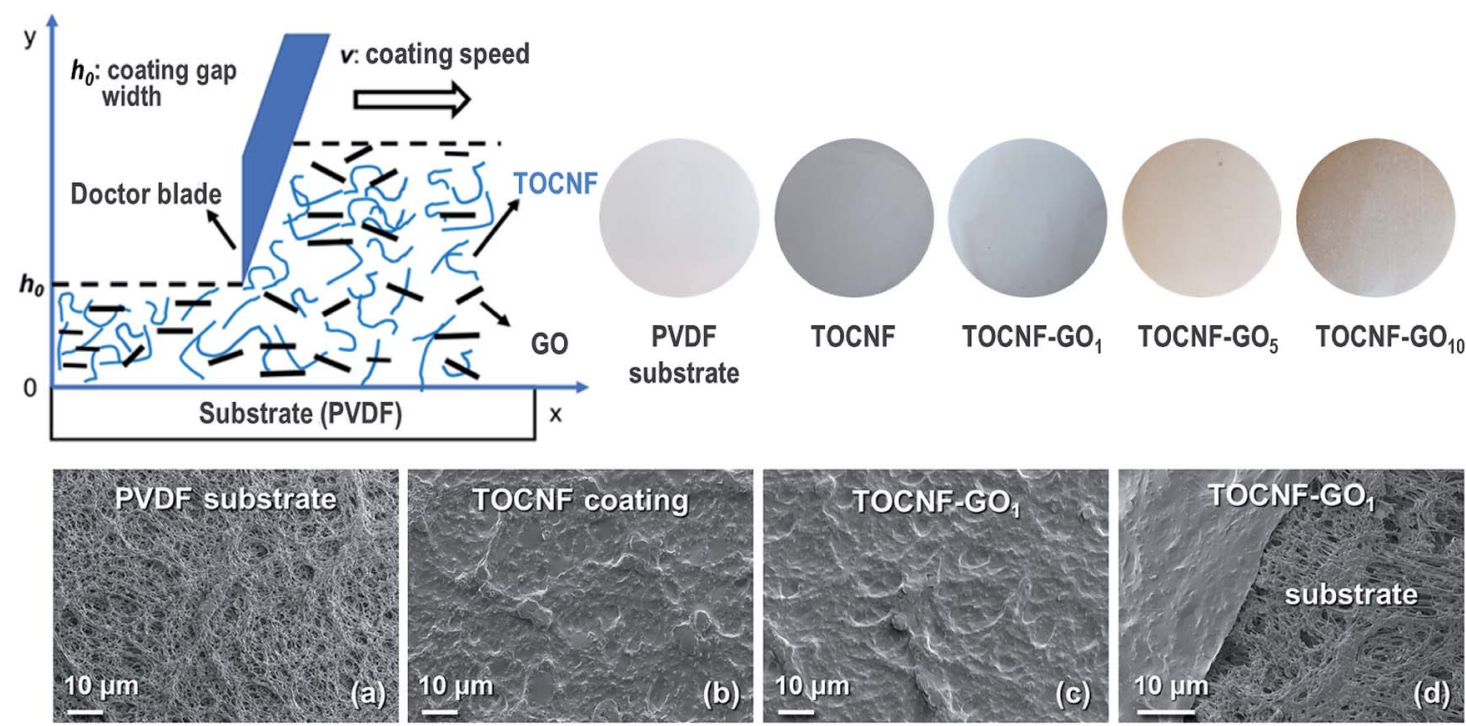

Fig. 3 Membranes with ultrathin coating layers fabricated using blade coating (schematic representation - top left). (Top right) photographs of the PVDF substrate, the TOCNF matrix and the TOCNF-GO coated membranes. (Bottom) SEM images of the porous PVDF substrate (a), the TOCNF coated surface (b), the TOCNF-GO ${ }_{1}$ coated surface (c), and a cross-section of TOCNF- $\mathrm{GO}_{1}$ coated membrane (d).

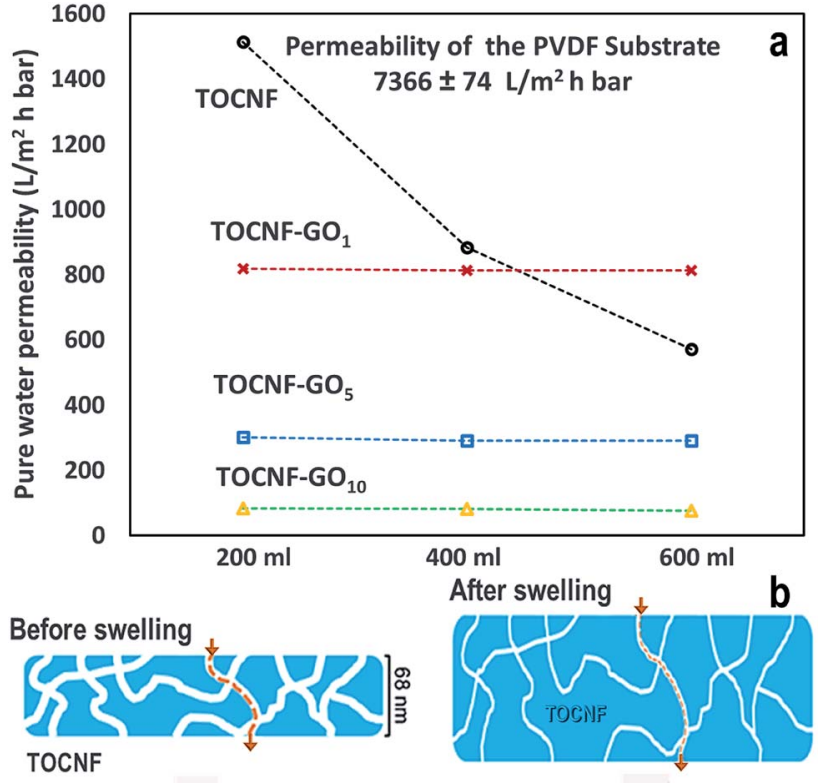

Embedded GO nanosheets bring about swelling resistant property

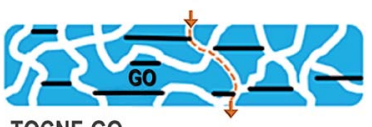

TOCNF-GO

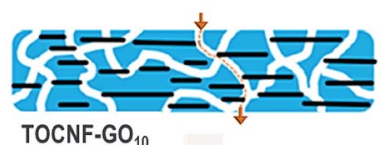

TOCNF-GO

Fig. 4 (a) Water permeability derived from three consecutive permeability measurements with the same conditions. (b) Schematic view for percolation through the ultrathin coated film.

filtration, or casting, had their thickness in a range of dozens of microns and simply exhibited no water permeability. ${ }^{64}$ It was reported, in fact, that for a $20 \mu \mathrm{m}$ thick TOCNF film with excellent gas barrier properties, the oxygen permeability was $1 \mathrm{~mL}$ per $\mathrm{m}^{2}$ per day per $\mathrm{Pa}^{21,62}$ To the best of our knowledge,

this is the first study describing the preparation of highly permeable TOCNF based membranes by means of the doctor blade coating technique. In addition, this study also found that the addition of $1 \mathrm{wt} \%$ GO could greatly inhibit swelling and packing of the membranes, which is a major improvement in relation to TOCNF membranes. Given these results we opted for the TOCNF- $\mathrm{GO}_{1}$ membrane for further characterizations.

\subsection{Dye retention performance}

3.5.1 Effect of hydrated radius and charge of the dyes. The effects of the charge and hydrodynamic/hydrated radius (radius of an equivalent hard sphere diffusing at the same rate as the molecule under observation) of the $\mathrm{VB}, \mathrm{RB}, \mathrm{MO}, \mathrm{R} 6 \mathrm{G}, \mathrm{MB}$ and $\mathrm{MnB}$ dyes on the retention of TOCNF- $\mathrm{GO}_{1}$ membrane $(68 \mathrm{~nm})$ and its porous substrate (pore size approximately $0.45 \mu \mathrm{m}$ ) were evaluated and are displayed in Fig. 5 . The retention $v s$. hydrated radius of the dyes (Fig. 5(a)) reveals that the TOCNF-GO membrane was more efficient than the PVDF support in retaining the dyes, irrespective of their size and charge. This is because, PVDF is a hydrophobic polymorphic fluoropolymer with amorphous and crystalline regions and, as such, does not exhibit selective adsorption due to the low polarizability and the strong electronegativity of the fluorine atoms. In contrast, TOCNF-GO matrices contain several carboxyl, hydroxyl and epoxy moieties that impart a negatively charged character to the exposed interfaces (thus a preferential binding of positively charged molecules) and are actively involved in intermolecular interactions stabilizing the material. These groups are also fundamental to the entrapment of local external agents present in the surrounding environment. Additionally, the GO content mitigates the hydrophilicity of the TOCNF-GO membranes since the large surface area of the sheets, with their decorating groups, are amphiphilic systems that can be involved in direct 

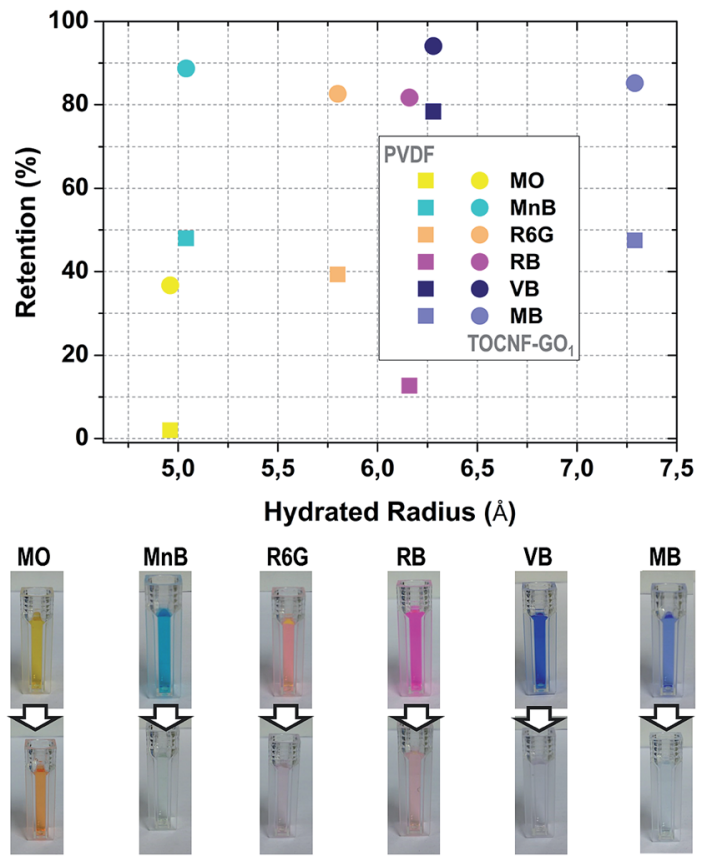

Fig. 5 (Top) Retention performance of TOCNF- $\mathrm{GO}_{1}$ membrane as a function of hydrated radius for the probe dyes (feed concentration: $10 \mathrm{mg} \mathrm{L}^{-1}$, each measurement was repeated three times). The hydrated radius of $\mathrm{MO}, \mathrm{RB}, \mathrm{MB}, \mathrm{MnB}^{34}$ and $\mathrm{R} 6 \mathrm{G}^{65}$ was found in the literature. The hydrated radius of $V B$ was estimated from SASA calculations; (Bottom) photos of feed and permeate solutions filtered through TOCNF-GO 1 membrane.

$\pi$-stacking interactions with themselves or with the hydrophobic portions of the approaching solutes. As a consequence, the TOCNF- $-\mathrm{GO}_{1}$ membrane had the best retention (>80\%) of all the positively charged molecules, and of all the molecules with a hydrated radius greater than $5 \AA$ irrespective of their supposed charge, as confirmed by visual examination of the permeate solutions (Fig. 6(b)). Rejection was in the following order: $\mathrm{MO}(-)<\mathrm{RB}(0)<\mathrm{R} 6 \mathrm{G}(+)<\mathrm{MB}(-)<\mathrm{MnB}(+)<\mathrm{VB}(+)$. The high retention of $\mathrm{MB}$ could be explained by considering the cooperative favourable interactions of its rings with the hydrophobic part of the support and the local neutralization effects of the $\mathrm{SO}_{3}{ }^{-}$groups of dye by hydrogen exchanges with the surrounding environment. The high retention of VB (94\%), where the estimated hydrated radius is around $6.3 \AA$, seems due to the combination of its positively charged character, the limited flexibility of its structure and to the favourable interactions of the rings with the support (most probably with the GO sheet planes). The formation of molecular aggregates, observed in the simulation results, however, could increase the hydrated radius and further favour an improved retention. These data suggest that the molecular weight cut-off (MWCO) of the membrane could be around $0.5 \mathrm{kDa}$ (ref. 66) and the pore size around $1 \mathrm{~nm}$, which confirmed the identified nanofiltration range.

A closer inspection of the structure of the dyes (Fig. S1, ESI $\dagger$ ) evidenced that the presence of many conjugated rings reduced the flexibility of the molecules, and their whole size had only
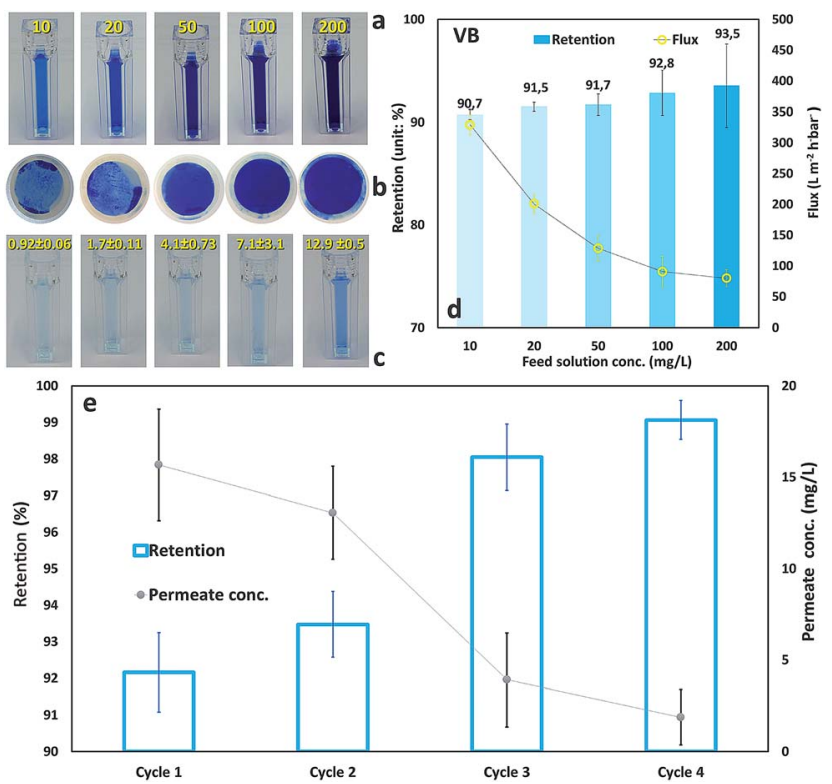

Fig. $6(a-d)$ Retention performance and the corresponding flux of TOCNF- $\mathrm{GO}_{1}$ membrane for VB at different feed concentrations; (e) retention performance and the corresponding flux of TOCNF-GO membrane for VB during four consecutive filtration tests.

negligible variation inside the small populations of conformers. A few more considerations regarding the dyes structures in relation to their shape could also be useful to identify possible correlations of these properties with the membrane retention mechanism. To this aim, the radius of gyration $\left(\mathrm{R}_{\mathrm{gy}}\right)$, which closely reflects the conformation of the dye and hydrated radius $\left(R_{\mathrm{h}}\right)$, which reflects the apparent size adopted by the solvated, tumbling, molecule, reported in Table S2 (ESI $\dagger$ ) can be considered representative values of each species. In Table $\mathrm{S} 2$ (ESI), $\dagger$ the ratio of $R_{\mathrm{gy}}$ and $R_{\mathrm{h}}\left(R_{\mathrm{gy}} / R_{\mathrm{h}}\right)$, which could provide shape information is also reported. It was observed, indeed, that globular structures had a characteristic ratio $R_{\mathrm{gy}} / R_{\mathrm{h}}$ around $0.78\left(R_{\mathrm{gy}}<R_{\mathrm{h}}\right){ }^{67}$ whereas in the case of non-spherical or elongated structures the ratio was higher $\left(R_{\mathrm{gy}}>R_{\mathrm{h}}\right)$. The ratios in Table S2 (ESI) $\dagger$ suggest that the rhodamines and Victoria blue B have almost spherical shapes, whereas the other three dyes are more elongated, which is consistent with the shapes shown in Fig. S1 (ESI). $\uparrow$ These features could be important in the design of the shape of the membrane pores especially in view of the dynamic tumbling of the molecules. To explain further the behaviour of the dyes during their adsorption on the supports, apart from their size, the electrostatic character was taken into account. In Fig. S2 (ESI), $\uparrow$ the MEP of R6G, which is a positively charged molecule $(+1)$, is displayed, on the top-right side of the figure, just to show the effect of the molecular charge on the MEP visualization. Thus, to identify the groups of this dye that could be involved in recognition and binding to the surface sites and disclose the nature of the possible interactions, two types of possible neutralization were simulated: (1) deprotonation of one of the nitrogens (top-left image); (2) deprotonation of both of the nitrogens and protonation of the ring oxygen. As can be noted, these two choices influence the whole charge 
distribution but a few features remain almost unaltered and independent of the modified site. These are the potential of the carbonyl oxygen, which is strongly negative, the negative potential on the planar conjugated rings, and the slightly positive potential of the side chains. Moving to RB, which is the only neutral molecule, similar areas are identified suggesting that the most effective binding mode could be through $\mathrm{H}$ bonding interactions between the carbonyl oxygen (and/or the $\pi$-density of the rings) and the hydroxyl groups of the surface complemented with the stacking of the dye and GO sheet rings. Regarding MB (Fig. S3, ESI $\dagger$ ), it is apparent that after neutralization the MEP becomes prevalently positive suggesting it favorable adsorption on the negatively charged portion of the TOCNF-GO support. On the contrary, MO shows a remarkable dipolar character with a dominant negative potential that suggests a weaker binding to the substrate, probably perpendicular to its surface. As far as the VB MEP is concerned, it could be speculated that the redistribution of the charge upon adsorption should favor flat orientations and formation of stacked aggregates.

3.5.2 Effect of feed concentration and membrane recycling. To investigate further the filtration performance of the TOCNF- $\mathrm{GO}_{1}$ membrane at different feed concentrations, its retention and solution flux of $\mathrm{VB}$ were estimated at feed concentrations in the range $10-200 \mathrm{mg} \mathrm{L}^{-1}$. Fig. 6(a) clearly shows the color variation between feed solutions and the corresponding permeates at all feed concentrations. Unexpectedly, the retention of TOCNF- $\mathrm{GO}_{1}$ membrane of VB slightly increased from $90.7 \%$ to $93.5 \%$ with the rising feed concentrations. The solution flux was drastically reduced at high feed concentrations partially because of the longer filtration pathway created by the thicker VB filter cake (Fig. 6(b)). It is also assumed that a part of the rejected molecules could be trapped in the membrane and partially occlude the pores. Therefore, a higher amount of rejected molecules at high feed concentration could cause pore narrowing. This is in line with the tendency of VB to form stacked aggregated structures. In general, commercially available membranes should guarantee an effective stable separation during filtering of large mass solutes. Therefore, filtration experiments at high feed concentration $\left(200 \mathrm{mg} \mathrm{L}^{-1}\right.$, $\mathrm{VB})$ were performed repeatedly four times on the same TOCNF$\mathrm{GO}_{1}$ membrane by measuring the retention and permeate concentrations. Fig. 6(e) shows that the retention continuously increased with the number of filtration tests, due to the pore size narrowing effect. The retention of VB at the fourth filtration was $99.1 \%$ and the permeate concentration was only $1.9 \mathrm{mg} \mathrm{L}^{-1}$. As reported in Table 1 , the dye weight rejected by TOCNF- $\mathrm{GO}_{1}$ membrane is 31.5 times higher than the mass of the coated barrier film. In summary, it was found that both GO nanosheets and TOCNF exhibited a high adsorption capacity of organic molecules because of their high specific surface area and functionality. The retention mechanism in TOCNF- $\mathrm{GO}_{1}$ membrane was more reliant on molecular size sieving rather than adsorption at high concentration, since the amount of dye rejected by the membrane was tens of times higher than its adsorption capacity (Table 1).
Table 1 VB retentate versus coating layer mass for VB during four consecutive filtration tests

\begin{tabular}{lllll}
\hline Coated film mass (mg) & 0.146 & & & \\
& Cycle 1 & Cycle 2 & Cycle 3 & Cycle 4 \\
Dye rejection mass $(\mathrm{mg})$ & 1.1 & 2.2 & 3.4 & 4.6 \\
Dye rejection capacity $\left(\mathrm{mg} \mathrm{g}^{-1}\right)$ & 7600 & 15300 & 23300 & 31500 \\
\hline
\end{tabular}

\subsection{MD simulation results}

3.6.1 Nanocellulose-based supports. As already observed in other computational studies and deduced from the experiments conducted during this work, the GO layer was stably adsorbed on the cellulose matrix and no diffusion took place over the whole simulation time period. This was mainly due to the relatively strong connections between the GO molecules and TOCNF fibers which were essentially multiple hydrogen bonds involving primarily the hydroxyls of the two species but also the carboxyl moieties. The dynamic nature of these types of interaction was responsible for the moderate mobility of the sheets and their relocation on top of the fibril facet. The type and entity of the rearrangements were determined further by GO selfinteractions, local morphology and packing of the fiber network. The most preferred orientations, in terms of molecule/ surface binding were those that maximized the contacts with the glucosyl units (realized through parallel alignments): oxygen-hydroxyl and hydroxyl-hydroxyl intermolecular hydrogen bonds were there reinforced by the participation of the carboxyl groups of GO and TOCNF. Other configurations with GO units inclined in relation to the fiber interfaces and slightly bent, were found to cross-link the different fibers and to create more porous regions. These local relocations, which were also supported by the flexibility of the cellulose chains, formed alternate porous and packed structure patterns. The negatively charged character attributed to the cellulose based support by experiment was confirmed by the examination of the charges of the atoms (EEM method) of the interface and their sums in the final configurations. In response to the perturbation due to dye adsorption and counterions, the entire matrices were negatively charged. This was also the case for the GO sheets, where the negatively charged character was mainly due to the presence of the carboxyl moieties. As far as solvation effects were concerned, the GO-TOCNF model had a slight tendency to inflate which was, however, remarkably reduced with respect to the models lacking in the GO component. ${ }^{68}$ This was confirmed by the increasing root mean squared deviations of the carbon atoms of the chains in relation to the more compact arrangements of the starting configuration. At the end of the production runs the values were stabilized around $8 \AA$.

\subsubsection{Dyes adsorption on the supports}

Radial distribution functions. The adsorption of the dyes on the substrate was confirmed by the presence of sharp peaks at short distances in the radial distribution functions between the atoms of the dyes and the oxygen atoms of the supports that were the principal contact points between the adsorbates and the interfaces (Fig. 7). Even though at the beginning of the simulations all the dyes were relatively far from the 
nanocomposite substrates and quite close to one another, the sampling time was sufficient to observe their migration, adsorption on the interfaces and their marked tendency to selfassemble.

Inspection of the VB RDF nitrogen plots in Fig. 7a-c indicates the involvement of these atoms in hydrogen bonding interactions with the hydroxyl groups of the supports when the GO sheets do not cover completely the fiber interface (Fig. 7c black peaks centered at around $2.8 \AA$ ), whereas when GO molecules laid on the TOCNF fiber, the nitrogens remained far from the cellulose matrix and were instead engaged in hydrogen bonding interactions with the adsorbed GO layers. In contrast, in more porous structures where the sheets were inclined in relation to the fiber, a number of hydrogen bonded structures appeared and included adsorption of the dyes on GO. Indeed, the magenta curve, visible in the RDF graphs, confirms the existence of VB-GO complexes, which were mainly realized through stacking or T-shaped interactions of the rings and hydrogen bonds. The peaks corresponding to the carboxyl groups of TOCNF were found, instead, at longer distances (broad peaks at about $4 \AA$ - Fig. 7c) when the GO sheets were inclined, whereas in the case of the GO flat adsorption a few interactions at short distances with the carboxyl atoms were noticed. As far as the solvation of the dye is concerned, the blue plots indicate water molecules directly hydrogen bonded to the VB nitrogens (peaks at $2.8 \AA$ ). These are present in both the simulations but when the structure was more packed, namely GO flat adsorption, the whole molecular arrangement prevented water from being directly connected to the dye. The second water shell was located at about $4.7 \AA$ and the solvent

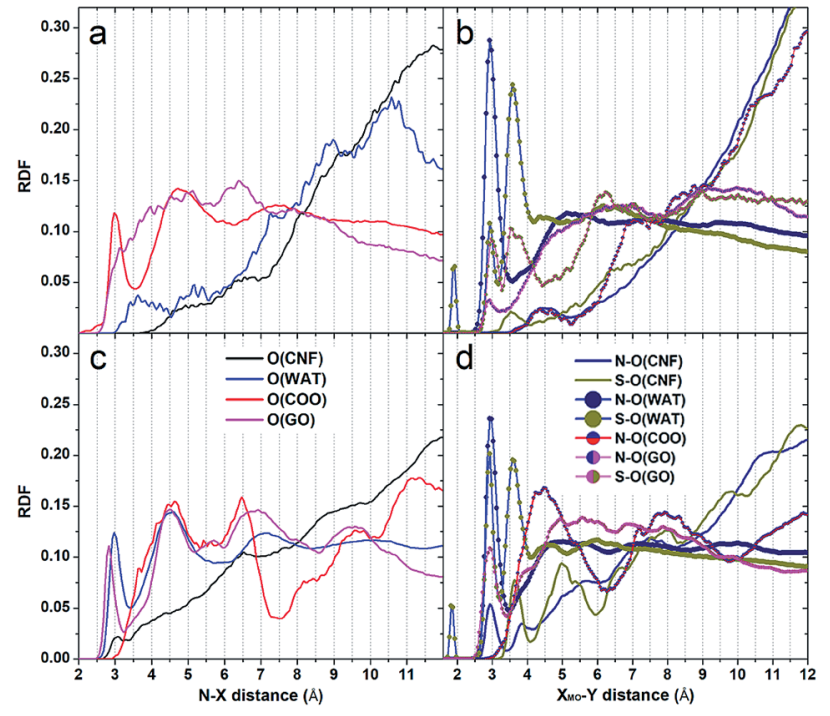

Fig. 7 (Left hand side) Normalized RDF of the adsorption of VB on GO-TOCNF. Only the nitrogen atom of the dye has been considered and its RDFs with all the oxygen types included in the systems have been calculated. (Right hand side) Normalized RDFs of the adsorption of MO. RDFs of nitrogen, sulfur and oxygen of MO with TOCNF oxygens, carboxyl oxygens, GO oxygens and water oxygens. ( $a$ and $b$ ) Sheet arrangements parallel to the fiber surface, ( $c$ and $d$ ) sheet arrangements inclined in relation to the fiber surface. was exchanged between the two shells. Moving to the MO RDFs, it was found that both the $\mathrm{N}$ atoms and the $\mathrm{SO}_{3}$ groups were well surrounded by water molecules displaying sharp peaks at around 2.8 and $3.8 \AA$, respectively, whereas considering the hydroxyl oxygen of TOCNF these distances were slightly longer (2.9 ̊̊ for N) and shorter (3.3 ̊̊ for S), respectively. Only $\mathrm{SO}_{3}$ was found close to the carboxyl groups (sharp peak at $3.6 \AA$ ), but not the nitrogen, which seemed located at around $5.1 \AA$. When TOCNF was covered with GO, the carboxyl groups were less exposed and the dyes were essentially adsorbed onto the GO planes even though, due to their size and shape, they could reach more easily than $\mathrm{VB}$ the hydroxyl groups of the TOCNF substrate. The shape and position of the peaks suggest that MO could adopt most probably vertical orientations relative to the support and be in touch with it just with one of the terminus moieties, primarily the $\mathrm{N}$ terminus. This agrees perfectly with the characteristic positive MEP potential of the molecule in that portion (Fig. S4 $\dagger$ ) and to the typical negatively charged tendency of the TOCNF interface. As far as the location of the $\mathrm{Cl}^{-}$and $\mathrm{Na}^{+}$ counterions are concerned, they were found prevalently close to the nitrogen atoms of $\mathrm{VB}$ and coordinated to the $\mathrm{SO}_{3}$ moiety of MO.

Spatial distribution functions. To complement the RDF description, three dimensional spatial distribution functions were also analyzed and used to identify the most populated locations of the nitrogen, sulfur and oxygen atoms of the dyes during the production dynamics. This was very useful to characterize more deeply their adsorption on the cellulose based support (Fig. S5-S8, ESI $\dagger$ ). A contour density 1.5 times larger than the average solvent density was chosen to reveal the most visited sites on the GO-TOCNF interfaces considering the last portion (100 structures) of the production trajectory. VB SDFs, shown in Fig. S5 and S6 (ESI), † indicate that in the case of a layer model (where the matrix is more compact - Fig. S5 $\dagger$ ), the dye molecules were stably adsorbed on the support ( $\mathrm{N}$ blue contours located close to the supports) in an almost flat orientation and they could interact with both the adsorbed GO molecules and small portions of the TOCNF fibers, remaining essentially bound to specific surface sites because of the entrapping effects at the sheets boundaries. Their rings were found mainly in stacking orientation with respect to the GO planes. Such a behavior could also be due to the redistribution of the negative charge on the substrate that perturbed not only the molecular orientation but also the locations of the counterions. Then, cooperative effects could drive the molecules towards different regions on the surface and also induce the migration of counterions into the solvent (green areas extending into the solvent). Comparison with the more porous model (Fig. S6, $\mathrm{ESI} \dagger$ ), where the GO sheets were inclined relatively to the fiber surface, revealed that the mobility of the dye, there, was markedly increased, due to their possible reorganization inside the created nanopore structures where solvent molecules are also present. A complete different scenario emerges, instead, from the inspection of the MO SDF profiles (Fig. S7 and S8, ESI $\dagger$ ). The main characteristic features of the MO adsorption were a marked tendency to self-assemble and to adopt a great variety of alignments relative to the support. This happened in solution 
and on the surface. Self-assembly was realized by means of stacking and T-shaped interactions of the rings and interconnection of the $\mathrm{SO}_{3}$ moieties with interposed $\mathrm{Na}^{+}$ions (mediators). This is clearly visible in Fig. S7 and S8. $\dagger$ The sparse distribution of the densities indicates greater mobility of the dye and a marked tendency to change frequently its orientation in relation to the support. The $\mathrm{SO}_{3}$ moieties could be found almost in touch with the interface when the $\mathrm{Na}^{+}$counterions were close by. However, the general tendency of MO to interact with the support through its terminus groups adopting different types of arrangement was confirmed. This was easily realized because of the size and shape of the molecule and the dipolar character of its MEP potential (Fig. S4, ESI $\dagger$ ). A similar scenario appears in the case of GO sheets in inclined orientations (Fig. S8, ESI $\dagger$ ). It is evident that the MO molecules could be entrapped among the sheets and the TOCNF surface; they could self-assemble but also cross-link the TOCNF chains through the mediating actions of the counterions. In summary, the simulations results suggest that the lower retention of MO could be due to a combination of dye properties: first its negatively charged character during the recognition process (driving force at long range when the molecule was far from the interface), and then, its strongly dipolar MEP when it was neutralized by the surrounding environment upon binding (counterions). Size, shape and mobility affected MO binding and retention on GOTOCNF as well. Indeed, comparison of the MEP of the other dyes, which seemed more stably adsorbed on the composite according to the retention data, suggests that more alternate positive-negative potential regions and the prevalence of positive MEP regions were preferred. In terms of molecular group, size and shape, the data suggested that condensed ring and branched conformations (such as the MB one) were more preferentially entrapped than the other more flexible species and that, besides the charge, these features played an important role in adsorption and retention.

\section{Conclusion}

In this study, we have successfully demonstrated that an ultrathin $(<100 \mathrm{~nm})$ TOCNF barrier film embedded with GO nanosheets can be built on a porous substrate by means of the doctor blade coating technique. Due to its nanoscale thickness and to the presence of GO nanosheets, the nanofiltration membrane exhibited high stability and excellent water permeability (816 \pm $3.4 \mathrm{~L} \mathrm{~m}^{-2} \mathrm{~h}^{-1}$ bar $^{-1}$ ), which had superior properties compared to previously reported nanocellulose based membranes. The new membrane showed an efficient and effective separation capability (including both high retention and permeation flux) of molecules with MWCO of $0.5 \mathrm{kDa}$ and revealed a stable filtration performance. The rejection mechanism of dyes was governed by size, shape, stacking, charge and concentration of the molecules in water solution, at optimised $\mathrm{pH}$ conditions. The TOCNF-GO ultrathin barrier film was capable of adsorbing and storing various types of molecular dyes with different characteristic structure and charge nature. The modeling studies revealed that the molecules could be stably adsorbed on the hybrid supports both through intermolecular hydrogen bond and stacking (or T- shaped) interactions of their rings with the GO planes. Dye selfassembly phenomena in solution and on the supports were also observed and suggested that even though the hydrodynamic radius of some dyes was too small to guarantee their entrapment, their aggregation tendency could disfavor their release in solution. Thus, retention could also be effective due to the formation of aggregate structures. In summary, we have designed a scalable eco-friendly charged membrane with good permeability for water purification that can be further extended to applications involving ion permeation.

\section{Conflicts of interest}

There are no conflicts to declare.

\section{Acknowledgements}

We acknowledge the financial support from Swedish Research Council (VR, grant no: 621-2013-5997 and 2017-04254) and Knut and Alice Wallenberg Foundation under Wallenberg Wood Science Centre (WWSC). We thank Fabian Andreas Araoz (Chemical Engineering and Technology, Royal Institute of Technology) for the film applicator (Electrometer 4340). SM is grateful to Adri C. T. van Duin for the standalone version of ReaxFF, for his collaboration and support. SM thanks the SCM experienced team for their technical support during the various stages of this investigation.

\section{References}

1 R. Xiong, H. S. Kim, S. Zhang, S. Kim, V. F. Korolovych, R. Ma, Y. G. Yingling, C. Lu and V. V. Tsukruk, ACS Nano, 2017, 11, 12008-12019.

2 X. Yang, Y. Du, X. Zhang, A. He and Z.-K. Xu, Langmuir, 2017, 33, 2318-2324.

3 R. V. Kandisa, K. V. N. Saibaba, K. B. Shaik and R. Gopinath, J. Biorem. Biodegrad., 2016, 7, 1000371.

4 V. Katheresan, J. Kansedo and S. Y. Lau, Chem. Eng. J., 2018, 7, 4676-4697.

5 D. Karisma, G. Febrianto and D. Mangindaan, IOP Conf. Ser.: Earth Environ. Sci., 2018, 195, 012057.

6 X. Peng, J. Jin, Y. Nakamura, T. Ohno and I. Ichinose, Nat. Nanotechnol., 2009, 4, 353-357.

7 S. J. Gao, H. Qin, P. Liu and J. Jin, J. Mater. Chem. A, 2015, 3, 6649-6654.

8 J. Wang, P. Zhang, B. Liang, Y. Liu, T. Xu, L. Wang, B. Cao and K. Pan, ACS Appl. Mater. Interfaces, 2016, 8, 6211-6218.

9 H. Ma, C. Burger, B. S. Hsiao and B. Chu, J. Membr. Sci., 2014, 454, 272-282.

10 M. J. Penna, M. Mijajlovic and M. J. Biggs, J. Am. Chem. Soc., 2014, 136, 5323-5331.

11 H. Wu, B. Tang and P. Wu, J. Membr. Sci., 2013, 428-433, 425. 12 H. Ting, H.-Y. Chi, C. H. Lam, K.-Y. Chana and D.-Y. Kang, Environ. Sci.: Nano, 2017, 4, 2205-2214.

13 H.-Y. Chi, S.-H. Hung, M.-Y. Kan, L.-W. Lee, C.-H. Lam, J.-J. Chen and D.-Y. Kang, CrystEngComm, 2018, 20, 54655474 . 
14 L. Yang, Z. Wang and J. Zhang, J. Membr. Sci., 2017, 532, 7686.

15 Q. Xie, S. Zhang, Z. Hong, H. Ma, B. Zeng, X. Gong, W. Shao and Q. Wang, Chem. Eng. J., 2019, 368, 186-201.

16 Q. L. Xie, W. Y. Shao, S. S. Zhang, Z. Hong, Q. Q. Wang and B. R. Zeng, RSC Adv., 2017, 7, 54898-54910.

17 G. S. Lai, W. J. Lau, P. S. Goh, A. F. Ismail, N. Yusof and Y. H. Tan, Desalination, 2016, 387, 14-24.

18 J. Wang, C. W. Zhao, T. Wang, Z. Wu, X. Li and J. S. Li, RSC Adv., 2016, 6, 82174-82185.

19 E. D. Smith, K. D. Hendren, J. V. HaagIV, E. J. Foster and S. M. Martin, Nanomaterials, 2019, 9, 125.

20 X. Cao, M. Huanga, B. Ding, J. Yu and G. Sun, Desalination, 2013, 316, 120-126.

21 A. Isogai, T. Saito and H. Fukuzumi, Nanoscale, 2011, 3, 7185.

22 H. Sehaqui, U. P. de Larraya, P. Liu, N. Pfenninger, A. P. Mathew, T. Zimmermann and P. Tingaut, Cellulose, 2014, 21, 2831-2844.

23 H. Ma, C. Burger, B. S. Hsiao and B. Chu, ACS Macro Lett., 2012, 1, 723-726.

$24 \mathrm{H}$. Voisin, L. Bergström, P. Liu and A. P. Mathew, Nanomaterials, 2017, 7, 57-76.

25 C. Zhu, P. Liu and A. P. Mathew, ACS Appl. Mater. Interfaces, 2017, 9, 21048-21058.

26 P. Liu, K. Oksman and A. P. Mathew, J. Colloid Interface Sci., 2016, 464, 175-182.

27 A. Mautner, H. A. Maples, H. Sehaqui, T. Zimmermann, U. P. de Larraya, A. P. Mathew, C. Y. Lai, K. Li and A. Bismarck, Environ. Sci.: Water Res. Technol., 2016, 2, 117-124.

28 S. Hokkanen, E. Repo, T. Suopajärvi, H. Liimatainen, J. Niinimaa and M. Sillanpää, Cellulose, 2014, 21, 1471-1487.

29 J. Wang and C. Chen, Biotechnol. Adv., 2009, 27, 195-226.

30 B. Volesky, Water Res., 2007, 41, 4017-4029.

31 R. Wang, S. Guan, A. Sato, X. Wang, Z. Wang, R. Yang, B. S. Hsiao and B. Chu, J. Membr. Sci., 2013, 446, 376-382.

32 K. W. Lawson and D. R. Lloyd, J. Membr. Sci., 1997, 124, 1-25.

33 H. Huang, Y. Ying and X. Peng, J. Mater. Chem. A, 2014, 2, 13772-13782.

34 A. Akbari, P. Sheath, S. T. Martin, D. B. Shinde, M. Shaibani, P. C. Banerjee, R. Tkacz, D. Bhattacharyya and M. Majumder, Nat. Commun., 2016, 7, 10891.

35 P. Sun, M. Zhu, K. Wang, M. Zhong, J. Wei, D. Wu, Z. Xu and H. Zhu, ACS Nano, 2013, 7, 428-437.

36 X. Wei, T. Huang, J. H. Yang, N. Zhang, Y. Wang and Z. W. Zhou, J. Hazard. Mater., 2017, 335, 28-38.

37 Q. Fang, X. Zhou, W. Deng, Z. Zheng and Z. Liu, Sci. Rep., 2016, 6, 33185.

38 M. S. Sajab, C. H. Chia, C. H. Chan, S. Zakaria, H. Kaco, S. W. Chook, S. X. China and A. M. Noor, RSC Adv., 2016, 6, 19819-19825.

39 T. Saito, S. Kimura, Y. Nishiyama and A. Isogai, Biomacromolecules, 2007, 8, 2485-2491.

40 M. J. Vainio and M. S. Johnson, J. Chem. Inf. Model., 2007, 47, 2462-2474.

41 J. S. Puranen, M. J. Vainio and M. S. Johnson, J. Comput. Chem., 2010, 31, 1722-1732.
42 M. J. Frisch, G. W. Trucks, H. B. Schlegel, G. E. Scuseria, M. A. Robb, J. R. Cheeseman, G. Scalmani, V. Barone, B. Mennucci, G. A. Petersson, H. Nakatsuji, M. Caricato, X. Li, H. P. Hratchian, A. F. Izmaylov, J. Bloino, G. Zheng, J. L. Sonnenberg, M. Hada, M. Ehara, K. Toyota, R. Fukuda, J. Hasegawa, M. Ishida, T. Nakajima, Y. Honda, O. Kitao, H. Nakai, T. Vreven, J. A. Montgomery Jr, J. E. Peralta, F. Ogliaro, M. Bearpark, J. J. Heyd, E. Brothers, K. N. Kudin, V. N. Staroverov, R. Kobayashi, J. Normand, K. Raghavachari, A. Rendell, J. C. Burant, S. S. Iyengar, J. Tomasi, M. Cossi, N. Rega, J. M. Millam, M. Klene, J. E. Knox, J. B. Cross, V. Bakken, C. Adamo, J. Jaramillo, R. Gomperts, R. E. Stratmann, O. Yazyev, A. J. Austin, R. Cammi, C. Pomelli, J. W. Ochterski, R. L. Martin, K. Morokuma, V. G. Zakrzewski, G. A. Voth, P. Salvador, J. J. Dannenberg, S. Dapprich, A. D. Daniels, O. Farkas, J. B. Foresman, J. V. Ortiz, J. Cioslowski and D. J. Fox, Gaussian 09, Revision A.02, Gaussian Inc., Wallingford CT, 2009.

43 A. Akbari, P. Sheath, S. T. Martin, D. B. Shinde, M. Shaibani, P. C. Banerjee, R. Tkacz, D. Bhattacharyya and M. Majumder, Nat. Commun., 2016, 7, 10891.

44 J. S. Murray and P. Politzer, Wiley Interdiscip. Rev.: Comput. Mol. Sci., 2011, 1, 153-163.

45 L. Liu, L. Miao, L. Li, F. Li, Y. Lu, Z. Shang and J. Chen, J. Phys. Chem. Lett., 2018, 9, 3573-3579.

46 P. Politzer and J. S. Murray, Rev. Comput. Chem., 2007, 2, 273-312.

47 G. S. Remya and C. H. Suresh, Phys. Chem. Chem. Phys., 2016, 18, 20615-20626.

48 Y. Okita, T. Saito and A. Isogai, Biomacromolecules, 2010, 11, 1696-1700.

49 A. K. Malde, L. Zuo, M. Breeze, M. Stroet, D. Poger, P. C. Nair, C. Oostenbrink and A. E. Mark, J. Chem. Theory Comput., 2011, 7, 4026-4037.

50 Z. Yanwu, M. Shanthi, C. Weiwei, L. Xuesong, J. W. Suk, J. R. Potts and R. S. Ruoff, Adv. Mater., 2010, 22, 3906-3924. 51 A. Paajanen and J. Vaari, Cellulose, 2017, 24, 2713-2725.

52 L. Yuanyuan, Z. Hongli, Z. Shuze, W. Jiayu, L. Zhen, O. Vaaland, S. Lacey, F. Zhiqiang, D. Hongqi and H. L. L. Teng, NPG Asia Mater., 2015, 7, e150.

53 N. V. Medhekar, A. Ramasubramaniam, R. S. Ruoff and V. B. Shenoy, ACS Nano, 2010, 4, 2300-2306.

54 J. L. Achtyl, R. R. Unocic, L. Xu, Y. Cai, M. Raju, W. Zhang, R. L. Sacci, I. V. Vlassiouk, P. F. Fulvio, P. Ganesh, D. J. Wesolowski, S. Dai, A. C. T. van Duin, M. Neurock and F. M. Geiger, Nat. Commun., 2015, 6, 6539.

55 S. K. Singh, S. G. Srinivasan, M. Neek-Amal, S. Costamagna, A. C. T. van Duin and F. M. Peeters, Phys. Rev. B, 2013, 87, 104114.

56 E. C. Neyts, A. C. T. van Duin and A. Bogaerts, Nanoscale, 2013, 5, 7250-7255.

57 SCM, Theoretical Chemistry, Vrije Universiteit, Amsterdam, The Netherlands, http://www.scm.com.

58 Z. Xu and C. Gao, ACS Nano, 2011, 5, 2908-2915.

59 R. Jalili, S. H. Aboutalebi, D. Esrafilzadeh, R. L. Shepherd,

J. Chen, S. Aminorroaya-Yamini, K. Konstantinov, 
A. I. Minett, J. M. Razal and G. G. Wallace, Adv. Funct. Mater., 2013, 23, 5345-5354.

60 B. Wang, W. Lou, X. Wanga and J. Hao, J. Mater. Chem., 2012, 22, 12859-12866.

61 F. C. Krebs, Sol. Energy Mater. Sol. Cells, 2009, 93, 394-412. 62 H. Fukuzumi, T. Saito, T. Iwata, Y. Kumamoto and A. Isogai, Biomacromolecules, 2009, 10, 162-165.

63 M. Hakalahti, M. Faustini, C. Boissière, E. Kontturi and T. Tammelin, Biomacromolecules, 2017, 18, 2951-2958.

64 A. Mautner, K.-. Lee, T. Tammelin, A. P. Mathew, A. J. Nedoma, K. Li and A. Bismarck, React. Funct. Polym., 2015, 86, 209-214.
65 I. I. Vlasov, A. A. Shiryaev, T. Rendler, S. Steinert, S.-. Lee, D. Antonov, M. Voros, F. Jelezko, A. Fisenko, L. F. Semjonova, J. Biskupek, U. Kaiser, O. L. Lebedev, I. Sildos, P. R. Hemmer, V. I. Konov, A. Gali and J. Wrachtrup, Nat. Nanotechnol., 2014, 9, 54-58.

66 L. Guo and P. H. Santschi, Environmental Colloids and Particles, 2007, pp. 159-221.

67 R. Scherrenberg, B. Coussens, P. van Vliet, G. Edouard, J. Brackman and E. de Brabander, Macromolecules, 1998, 31, 456-461.

68 C. Zhu, S. Monti and A. P. Mathew, ACS Nano, 2018, 12, 7028-7038. 